\title{
Protée
}

\section{Objets sans frontières}

\section{Andrea Semprini}

Volume 29, numéro 1, 2001

La société des objets. Problèmes d’interobjectivité

URI : https://id.erudit.org/iderudit/030611ar

DOI : https://doi.org/10.7202/030611ar

Aller au sommaire du numéro

Éditeur(s)

Département des arts et lettres - Université du Québec à Chicoutimi

ISSN

0300-3523 (imprimé)

1708-2307 (numérique)

Découvrir la revue

\section{Citer cet article}

Semprini, A. (2001). Objets sans frontières. Protée, 29(1), 9-16.

https://doi.org/10.7202/030611ar

\section{Résumé de l'article}

Cet article concerne l'analyse d'un type particulier d'objets, les « objets sans frontières ", dans le cadre d'une lecture sémiotique de la mondialisation. Après avoir rappelé les principales perspectives théoriques utilisées dans l'analyse des objets, nous y définissons les objets mondialisés et identifions leur spécificité dans le type de relation qu'ils entretiennent avec les contextes. Nous proposons ensuite une description du monde possible que ces objets projettent et de la dialectique d'interaction et de renforcement mutuel qui s'établit entre monde possible et monde réel. Cette dialectique montre que la mondialisation, loin d'être une question exclusivement économique, est aussi une affaire d’imaginaire et de représentations.
Ce document est protégé par la loi sur le droit d'auteur. L’utilisation des services d'Érudit (y compris la reproduction) est assujettie à sa politique d'utilisation que vous pouvez consulter en ligne.

https://apropos.erudit.org/fr/usagers/politique-dutilisation/ 


\section{O BJETS SAN S FRO NTIÈRES}
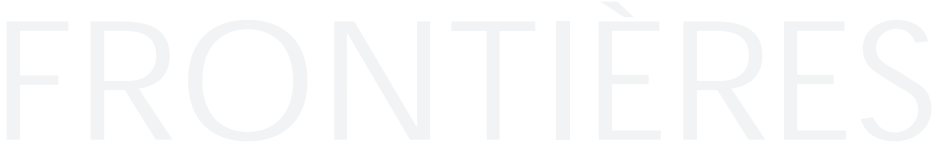

A N DREA SEMPRINI

\section{LA MONDIALISATION ET LES OBJETS}

Peut-on concevoir la mondialisation comme un immense système de signification, comme un mécanisme dont le cour et l'enjeu seraient non pas, ou non seulement, de type économique, mais essentiellement et plus

fondamentalement de type sémiotique? C'est l'hypothèse que nous allons explorer dans ces pages. Bien que très abstrait et pour tout dire presque insaisissable, le processus de mondialisation présente un aspect bien particulier et tout à fait concret: sa production très abondante d'objets, que l'on pourrait qualifier d'objets mondialisés. Dans les paragraphes qui suivent, nous allons présenter ces objets et développer quelques considérations à leur égard. À partir de cette analyse, des conclusions seront avancées sur le caractère sémiotique du processus de mondialisation ainsi que sur la place et le rôle des objets dans ce processus.

Avant d'en arriver aux objets mondialisés, il est tout de même nécessaire de s'attarder brièvement sur la notion de mondialisation. Ce concept - pas bien nouveau ni original, avouons-le - est revenu sur le devant de la scène depuis quelques années. Deux événements ont contribué à ce retour en force:

l'effondrement du bloc communiste en Europe, avec l'ouverture de nombreux pays à l'économie de marché, et le retour de la croissance économique un peu partout dans les pays industrialisés. La faillite des économies planifiées semble avoir confirmé, a contrario, la supériorité de l'économie libérale et capitaliste, et le retour de la croissance a provoqué une augmentation et une accélération des échanges économiques et des flux financiers. C'est dans ce cadre de lecture, de type éminemment économique, que la notion de mondialisation est revenue au centre du débat dans plusieurs pays.

Comme toutes les notions controversées et au centre d'enjeux internationaux, la mondialisation tend à polariser les positions entre partisans et opposants, voire résistants. Cette polarisation s'est doublée, dans le cas de la mondialisation, d'une opposition de type social, qui semble sortie tout droit du XIXe siècle, entre élite rapace et bon peuple naïf et inévitablement manipulé et exploité. Les incidents de Seattle, en novembre 1999, à l'occasion d'un sommet de l'Organisation mondiale du commerce, ont été une illustration parfaite de cette polarisation: les élites 
politiques, économiques et financières d'un côté, les étudiants, les représentants du tiers-monde, les minorités, les paysans et les citoyens ordinaires de l'autre. Les unes soutiennent mordicus les bienfaits de la mondialisation pour tous: davantage de choix, des marchandises moins chères, la possibilité de trouver les mêmes produits partout. Les autres ne voient dans la mondialisation que des menaces: la standardisation des produits, des aliments de mauvaise qualité, le triomphe des multinationales aux dépens des petits producteurs.

Ces positions sont a priori inconciliables, mais se rejoignent néanmoins sur un point fondamental: le fait de mettre des phénomènes économiques au centre du processus de mondialisation. Or sans vouloir remettre en cause ce qui semble relever de l'évidence même, à savoir l'importance de l'économie dans le processus de mondialisation (comme dirait l'autre: «It's the economy, stupid»), nous aimerions problématiser et nuancer cette évidence, et suggérer que l'économie n'est qu'un des aspects de la mondialisation, sans doute même pas le plus important. Les objets vont nous aider dans cette tâche.

La diffusion mondiale des marchandises ne date pas d'aujourd'hui. Elle fut même au cœur du développement de l'économie capitaliste, notamment dans sa phase d'expansion coloniale. Mais jusqu'à une époque relativement récente, disons les années 80 du $\mathrm{XX}^{\mathrm{e}}$ siècle, la mondialisation des marchandises était un phénomène essentiellement industriel et commercial. Ce n'est que depuis une petite vingtaine d'années que les objets qui déferlent partout dans le monde sont devenus autre chose que de simples marchandises, que des objets de consommation tout court. Ils portent avec eux une identité, des significations, ils incorporent des systèmes de représentations. Prenons quelques exemples concrets de ces objets emblématiques d'une certaine modernité consumériste: les hamburgers, avec l'incontournable panoplie du grand gobelet de soda et de la dose de frites; les chaussures de sport, dont les couleurs, les matériaux ou les inscriptions peuvent varier beaucoup, mais qui gardent une identité visuelle bien particulière, tous modèles confondus; les jeans et les tee-shirts, qui constituent d'ailleurs, avec les chaussures de sport, une sorte de syntagme obligé pour les jeunes du monde entier; les baladeurs; les téléphones portables, etc.

Cette liste pourrait continuer (consoles de jeux, ordinateurs portables, sac à dos, etc.), mais elle permet déjà d'avancer quelques considérations. Ces objets ont tendance à fonctionner par ensembles, à constituer des systèmes cohérents. Lorsqu'on en trouve un, les autres ne sont généralement pas bien loin. Ce sont pour la plupart des objets qui s'adressent à un public jeune (même si cette jeunesse apparaît davantage comme un état d'esprit que comme une réalité biologique). Ces objets relèvent de pratiques et d'un style de vie nomades, ou tout au moins extradomestiques. Enfin, et c'est le point qu'il nous importe de souligner ici, ils justifient pleinement l'appellation d' "objets sans frontières", en raison de leur diffusion et de leur consommation mondialisées.

Ces objets présentent donc un nombre de caractéristiques communes suffisantes pour être considérés comme formant une véritable "société d'objets", dans la mesure où ils partagent des règles de fonctionnement, des logiques d'usages, un public homogène et un style de vie commun. Avant d'aller plus loin dans l'analyse de cette famille si particulière d'objets, il est toutefois utile de rappeler rapidement l'«état de l'art» en matière d'analyse d'objets.

\section{CINQ APPROCHES}

DE L'ANALYSE SÉMIOTIQUE DES OBJETS

Nous pouvons identifier cinq approches principales relativement à l'analyse sémiotique des objets. Ces approches ne sont pas mutuellement exclusives, mais présentent une histoire, des matrices théoriques et un pouvoir heuristique différents.

\section{A. Analyse contextuelle}

Il s'agit de l'approche la plus connue et la mieux établie, en raison, entre autres, de sa proximité avec ce que les cognitivistes appellent l'«attitude naturelle». 
On peut faire remonter à Roland Barthes ${ }^{1}$ et à Jean Baudrillard ${ }^{2}$ le développement de ce type d'analyse, essentiellement d'inspiration sémiologique. Dans cette perspective théorique et méthodologique, le contexte (sémiotique ou autre), l'environnement qui entoure l'objet, joue un rôle primordial dans sa signification, qui est dès lors appréhendée sous forme de connotation, ou de système de connotations. L'objet ne signifie que dans la mesure où il est capable de recycler et en quelque sorte de "parasiter" les significations stéréotypées mises à disposition par son environnement. Ce type d'approche porte à privilégier les effets de système et à diluer la spécificité signifiante de l'objet, qui se trouve réduite à un effet de contexte. Cet angle de lecture tend également à sous-estimer les propriétés dynamiques de la signification de l'objet et sa capacité à intervenir sur le contexte.

\section{B. Analyse immanente}

Très en vogue dans les années 70 et 80 en raison de son ascendance structuraliste, ce type d'analyse tend à se concentrer sur l'objet lui-même. Elle s'attache à décrire, sur le modèle de l'analyse sémionarrative des textes, le processus qui permet à l'objet de manifester sa signification. On pense d'ailleurs que cette dernière est en grande partie inscrite dans les propriétés textuelles de l'objet et que la démarche analytique a pour but de la rendre explicite. La source théorique canonique pour ce type d'approche est sans conteste le modèle sémio-narratif d'A.J. Greimas ${ }^{3}$. Si elle permet d'étudier de manière très fine le processus de signification immanent à l'objet, cette démarche tend à négliger le rôle du contexte et la variété des formes de réception. Elle tend également à considérer la dimension textuelle de l'objet comme une donnée non problématique.

\section{Analyse intersubjective}

On peut considérer cette approche comme une tentative de dépasser les faiblesses des analyses contextuelles classiques ( $c f . \S 1)$. L'accent est mis ici sur la relation dynamique et mutuellement déterminante qui lie l'objet à son contexte. Ce dernier ne préexiste pas à l'objet, il émerge parallèlement à celui-ci, dans un processus qui est éminemment ouvert ${ }^{4}$. La signification de l'objet ne saurait être pensée ni comme une collection de signes à décoder (approche contextuelle classique), ni comme une donnée textuelle à dévoiler (approche immanente classique). Pour apprécier la dynamique d'émergence intersubjective des significations, cette démarche insiste également sur les conditions pratiques d'utilisation des objets. On peut assigner la paternité de ce type d'analyse aux ethnométhodologues 5 et aux sociologues des techniques ${ }^{6}$. Les limites de l'analyse intersubjective reposent essentiellement sur son parti pris microsociologique, qui empêche de dégager la signification de l'objet du contexte de son émergence.

\section{Analyse interobjective}

Tout comme le modèle intersubjectif représente un prolongement et une évolution du modèle contextuel, de la même manière on peut considérer la perspective interobjective comme un enrichissement de la perspective immanente. Cette démarche étant au cœur des contributions de ce numéro de Protée, nous ne nous y attarderons pas ici. Nous nous limiterons à souligner que la lecture interobjective déplace la question du contexte, en l'intégrant en quelque sorte à l'objet lui-même, sous forme de relation avec d'autres objets. Il s'agit dans un certain sens d'une tentative d' «immanentiser» le contexte, afin d'en tenir davantage compte que dans les approches immanentes classiques, sans pour autant renoncer aux avantages et à la finesse de l'analyse sémiotique canonique ${ }^{7}$. La richesse et la variété des contributions recueillies dans ce numéro témoignent de l'intérêt et du potentiel de cette démarche.

\section{E. Analyse sociosémiotique}

Cette perspective est une tentative de synthèse des deux précédentes, dont elle cherche à optimiser le potentiel. De l'analyse intersubjective, la démarche sociosémiotique retient la préoccupation pour les pratiques d'usage et la prise en compte des grammaires de réception et de la coopération des acteurs sociaux 
dans la production des significations ${ }^{8}$. De l'analyse interobjective, la sociosémiotique retient la démarche objectivante et l'ambition généralisante, à savoir le fait de dépasser une perspective exclusivement microsociologique ou microsémiotique. En propre, elle introduit une préoccupation spécifique pour la dimension culturelle des significations étudiées. Celles-ci ne sont pas considérées, contrairement à ce qui est souvent le cas dans les analyses sémiotiques, comme de simples contenus dont on se contente de dégager la valeur conceptuelle. Elles sont insérées dans le processus de diffusion, de circulation et de réception des discours sociaux et elles sont rattachées aux valeurs et aux symboliques sociales exprimées par des acteurs ou par des groupes d'acteurs sociaux ${ }^{9}$. Ce type d'analyse s'inscrit donc dans la tradition théorique des études culturelles.

\section{Des objets SANS CONTEXTE?}

Ces rappels nous permettent maintenant d'aborder l'analyse des objets mondialisés et d'introduire d'emblée une distinction qui montre la centralité de la question du contexte. Habituellement, un objet ne "fonctionne», c'est-à-dire ne peut produire ou véhiculer du sens, qu'à l'intérieur d'un environnement sémiotiquement cohérent. Ceci est tout aussi vrai pour les objets «traditionnels" que pour les objets de consommation. La prise en compte de ce contexte lors de l'analyse peut être différente selon la perspective théorique adoptée, mais il ne fait pas de doute que, dans les pratiques sociales réelles, un objet doit normalement s'inscrire dans un contexte pertinent. Pensons à l'effet incongru, voire surréaliste, engendré par une tranche de pizza présentée dans un repas protocolaire dans une ambassade, par un robot ménager installé à la caisse d'un cinéma ou par un parfum de luxe déposé dans de la litière pour chats. La pratique artistique a d'ailleurs rapidement compris la force du lien conventionnel qui lie un objet à son environnement. Depuis les ready-made de Duchamp, la décontextualisation et/ou recontextualisation des objets est devenue une forme d'expression artistique bien établie.

Mais si ces formes de déplacement artistique sont possibles, c'est justement parce que dans des conditions d'énonciation normales, les objets ne prennent tout leur sens qu'à l'intérieur d'un contexte. Nous approchons alors une caractéristique fondatrice des objets mondialisés, c'est-à-dire leur capacité de s'affranchir de l'environnement, de fonctionner comme si l'environnement socioculturel n'était pas ou plus pertinent. Les objets mondialisés semblent fonctionner soit de manière acontextuelle, en deçà de toute inscription dans un contexte local, soit de manière transcontextuelle, au-delà de tout contexte local. Dans le premier cas de figure, l'objet mondialisé semble être en mesure de flotter dans une sorte d'espace-temps qui lui serait propre et qui se passe de tout ancrage dans la réalité locale. Dans le second cas de figure, il semble capable de cohabiter avec le contexte local, sans vraiment l'intégrer, mais sans le remettre radicalement en discussion non plus. Il négocie une sorte d' "entente cordiale" qui lui permet de s'insérer dans un contexte sans en modifier les paramètres de base.

Bien que ces acontextualités ou transcontextualités continuent de nous surprendre et de nous paraître incongrues, elles permettent à l'objet mondialisé de se faufiler dans les moindres plis des usages sociaux, de joindre les groupes les plus éloignés, de s'insérer dans les contextes les plus divers. Les médias aiment nous surprendre en nous montrant des images du bédouin sur son chameau en plein désert avec son téléphone portable pendu à l'oreille, du jeune Africain en pleine brousse avec telle marque de chaussures de sport aux pieds, du paysan chinois croquant joyeusement un hamburger plein de sauce. Mais l'effet ready-made ne fonctionne plus. Ces associations, bien que peu probables, ne nous surprennent plus. Nous avons appris à reconnaître cette famille d'objets et à nous faire à leur extraordinaire élasticité par rapport aux contextes. C'est même cette flexibilité qui permet à un objet d'obtenir ce statut d'objet mondialisé.

\section{LE MONDE POSSIBLE DES OBJETS MONDIALISÉS}

Les exemples d'objets mondialisés donnés en ouverture (hamburger, chaussures de sport, tee-shirt, jeans, baladeur, téléphone portable, consoles de jeux, 
sac à dos) peuvent alors être considérés comme les occurrences d'un paradigme unique, d'une société d'objets, comme diraient E. Landowski et G. Marrone. Malgré leurs différences substantielles -d'usage, de fonction, de prix-, ces objets partagent un grand nombre d'aspects communs et peuvent être considérés, et analysés, comme un véritable système sémiotique.

Prenons par exemple la dimension formelle de ces objets, leur plan de l'expression. On remarquera qu'un certain nombre de formants se retrouvent dans presque tous ces objets. Des formes arrondies, des galbes rassurants, des couleurs claires, des matériaux doux et agréables au toucher. Même les objets les plus technologiques, comme les téléphones ou les ordinateurs portables, ont dû abandonner leurs livrées austères, grises ou noires, en faveur de couleurs gaies, de matériaux transparents, translucides. Le petit pain qui sert de base à la fabrication du hamburger -le bun - est à lui seul un résumé parfait de cette esthétique régressive, maternante et rassurante (niceness). Certains l'on comparé à un sein féminin, d'autres ont rapproché son goût sucré et sa matière spongieuse, qui se dissout rapidement dans la bouche, à la bouillie dont raffolent les nourrissons.

Nous pouvons intégrer à l'analyse d'autres propriétés de cette famille d'objets. Quatre dimensions nous semblent particulièrement pertinentes. En premier lieu, tous ces objets instaurent un rapport au corps particulièrement étroit, voire intime. Cette intimité entre en résonance directe avec la sphère sensorielle de l'individu. Ils peuvent viser la bouche et donc le goût (le hamburger); l'oreille et l'ouie (baladeur, téléphone); l'œil et la main, et donc la vue et le toucher (écran, souris, clavier d'ordinateur); le pied ou le corps tout entier (chaussures de sport, vêtements). Deuxièmement, la plupart de ces objets visent l'individu, sont pensés et conçus pour un usage et une jouissance individuels, voire solitaires (baladeur, consoles de jeux, hamburger). Cet aspect est directement relié au précédent. L'étroite imbrication de ces objets avec le corps souligne à la fois leur destination individuelle et le fait qu'ils s'adressent à la dimension corporelle de l'individu, à la personne. De manière significative, les utilisateurs entretiennent un rapport privé et personnel avec ces objets. On prête à la rigueur sa maison ou sa voiture, beaucoup plus rarement son portable ou ses chaussures de sport (et jamais son hamburger!)

Le troisième aspect, évoqué en ouverture, concerne la dimension nomade de la majorité de ces objets, le fait qu'ils sont conçus pour être consommés ou utilisés à l'extérieur de l'enceinte domestique, ou carrément en déplacement. Leur proximité au corps implique une miniaturisation qui permet de les transporter sans difficulté. La plupart de ces objets tiennent d'ailleurs dans une poche. Enfin, il est important de souligner que tous ces objets peuvent être utilisés soit dans une logique instrumentale (pour travailler, pour apprendre, pour s'informer), soit dans une logique de loisir (pour s'amuser, pour passer le temps, pour se distraire). Dans ce double mode, c'est l'utilisation «loisir» qui tend à être privilégiée, la première logique fonctionnant souvent comme alibi pour la seconde.

Ces différents éléments permettent - quatrième et dernier aspect qui synthétise d'ailleurs les précédentsde dégager un univers sémiotique assez clair et cohérent. Les objets mondialisés, considérés dans leur ensemble, considérés comme un paradigme unifié, construisent un monde possible aux contenus assez précis. Il s'agit d'un monde rassurant et vaguement régressif, d'où tout stress et tout principe de responsabilité ont été évacués. Un monde dominé par la facilité et le divertissement, où même le travail devient agréable et où les frontières entre vie professionnelle et loisirs tendent à s'estomper. C'est encore un monde d'individus libérés de toute attache sociale ou culturelle et libérés en particulier des contraintes du temps et de l'espace. Des individus nomades, en mouvement perpétuel, au gré des envies et des opportunités à saisir. Ce monde est dominé par le principe de plaisir et par la poursuite de l'épanouissement personnel. Le corps et les sens y ont une place de choix et alimentent le rêve d'une vie entièrement vécue à l'enseigne de l'hédonisme. Enfin, c'est un monde qui attribue la plus grande 
importance à la dimension expressive de l'individu, à la possibilité, érigée presque en droit (et peut-être même en devoir), de cultiver la créativité, l'intuition, la libre expression de soi.

\section{Monde POSSIBLE ET EFFICACITÉ SÉMIOTIQUE}

Ce système symbolique est extrêmement cohérent et puissant. Il construit un espace socioculturel qui est celui de la modernité attrayante, de l'hédonisme, de la consommation, de la communication, du bien-être individuel. Comment s'étonner si son pouvoir de séduction auprès du public, et notamment du public jeune, est considérable? Tout en étant un monde possible, une construction symbolique, ce système de représentations entretient des relations évidentes avec le monde "réel", avec la réalité sociale des différents contextes où il est présent. En paraphrasant la formule de Lévi-Strauss ${ }^{10}$, on pourrait définir ces relations en termes d'«efficacité sémiotique». Le monde possible engendré par les objets mondialisés ne se limite pas à déployer un espace de rêve ou de plaisir qui demeurerait séparé des activités et des expériences quotidiennes. Au contraire, il entre dans une relation dialectique avec l'environnement social, qu'il contribue à façonner, mais par lequel il est également façonné en retour.

En d'autres termes, on peut dire que les objets mondialisés permettent la projection (dans un premier temps exclusivement symbolique) d'un style de vie et d'une culture particulière, de type acontextuel ou transcontextuel. Dans la mesure où elle est adoptée, en totalité ou en partie, cette projection se transforme en style de vie ou en culture «réels». Ces derniers évoluent sous la poussée d'une infinité d'autres facteurs, tant endogènes qu'exogènes, et à leur tour finissent par faire évoluer le système symbolique qui était en surplomb. S'enclenche alors un processus continu d'influence mutuelle et de transformation permanente. Cette logique de fonctionnement, cette interaction permanente entre monde possible et monde réel n'est pas propre aux seuls objets mondialisés. Elle peut être identifiée également dans l'univers des médias et des industries culturelles mondialisées (CNN, BBC, Hollywood, publicités, marques). Mais dans le cas des objets, elle semble être particulièrement puissante, et ce pour une raison assez simple.

Les objets mondialisés (et d'ailleurs les objets tout court) ont en effet cette caractéristique de participer, d'emblée, des deux mondes, le monde possible et le monde réel. Contrairement à la plupart des dispositifs sémiotiques qui projettent l'espace socioculturel d'un monde possible mondialisé, les objets sont à la fois des constructions sémiotiques et des énoncés du monde naturel, des produits tout à fait concrets. On les mange, on les porte, on les manipule, on s'en sert. Ce sont donc des acteurs qui ont un impact non seulement sur nos représentations, mais également sur nos comportements et sur notre manière de vivre. C'est cette caractéristique, c'est cette double nature, sémiotique et fonctionnelle, symbolique et pragmatique, qui fait d'eux un phénomène particulièrement intéressant dans l'analyse des processus de mondialisation.

Nous pouvons maintenant poser la question de l'interobjectivité à l'intérieur de cette logique de fonctionnement si particulière. Comme on vient de le voir, le monde possible engendré par les objets mondialisés est homogène et systématique. Il propose un modèle paradigmatique fortement intégré. Sa projection dans le monde réel tend alors à construire des chainnes syntagmatiques ouvertes, où le dernier élément de la chaîne crée les conditions et la nécessité pour l'adjonction de l'élément suivant. Prenons comme exemple la question de la mobilité et du nomadisme. Certains objets (notamment le baladeur, le téléphone et l'ordinateur portables) ont fortement contribué à la construction du nomadisme comme caractéristique et comme valeur fondatrice d'un style de vie mondialisé. En même temps, le monde possible et les usages proposés par ces objets n'auraient pas trouvé un public si le style de vie nomade n'était pas déjà inscrit, en quelque sorte, dans l'évolution des comportements réels. Nous sommes donc typiquement dans le mécanisme d'efficacité sémiotique et d'influence mutuelle décrit plus haut. 
Mais une fois que le nomadisme existe comme paradigme sémiotique et comme suite syntagmatique d'objets réels (ceux qu'on vient d'évoquer), d'autres objets viendront prolonger, de manière presque naturelle, cette chaîne: l'organiseur, l'appareil photo ou la mini-caméra numériques, le baladeur MP3, etc. Ces objets trouvent leur pertinence dans le monde possible qui fait du nomadisme et de la mobilité permanente une valeur en soi dans le cadre du style de vie mondialisé; et ils trouvent en même temps leur cohérence dans le monde réel des comportements nomades, où ils occuperont une place et présenteront une utilité «réelles». On peut dire que ce mécanisme, une fois mis sur orbite, c'est-à-dire une fois qu'il a atteint sa vitesse de croisière, tend à s'auto-alimenter. L'arrivée dans le monde réel de chaque nouvel objet s'inscrit dans les comportements réels et renforce le monde possible qui lui a permis d'exister. En même temps, il prépare la place, en creux, pour un nouvel arrivant, qui va à son tour renforcer le système, dans un processus sans fin.

\section{Mondialisation et BRICOlage}

L'efficacité et la puissance de ce processus en inquiètent plus d'un. Le "succès» et la rapidité de la diffusion planétaire des objets mondialisés font craindre l'homogénéisation des cultures et, parallèlement, la perte de l'authenticité des identités et des styles de vie locaux. Cette crainte s'insère dans un débat plus large et très actuel sur les dangers de la mondialisation. Sans entrer dans ce débat général, l'analyse que nous venons de développer nous permet de relativiser ces craintes d'uniformisation. Mais pour ce faire, nous devons discuter un dernier point, celui de la réception. Ceux qui s'inquiètent le plus de l'aplatissement des valeurs provoqué par la mondialisation tendent systématiquement à sousestimer le phénomène de la réception. Le fait d'avoir souligné la dimension sémiotique des processus de mondialisation nous permet au contraire de rappeler le rôle fondamental de la réception, tant en soi que comme volet incontournable dans le processus de la semiosis.
L'erreur de beaucoup de lectures de la mondialisation est de considérer le phénomène en question comme un processus énonciatif uniforme. Si cette uniformité est certainement très importante du côté de la production, il en va tout autrement pour la réception. Revenons aux objets mondialisés. Nous avons vu que, d'un point de vue théorique, leur spécificité consiste dans leur capacité à fonctionner de manière acontextuelle ou transcontextuelle. Cette capacité les rend particulièrement aptes à véhiculer un monde possible "sans frontières" et à contribuer activement aux processus de mondialisation. Mais à y regarder de plus près, nous sommes bien obligés de reconnaître que cette acontextualité ne concerne que la mise en circulation des objets. Leur réception, elle, est nécessairement inscrite dans des contextes, dans des logiques et dans des grammaires de réception locales. Pour utiliser une terminologie plus sémiotique, on pourrait dire que si l'énonciation de ces objets est acontextuelle, leur saisie est toujours, du moins en partie, contextuelle ${ }^{11}$.

Cette distinction commence aujourd'hui à être admise en ce qui concerne le fonctionnement des industries culturelles à diffusion mondialisée. On sait, car de nombreuses études l'ont montré et confirmé, que l'on ne consomme pas de la même manière Dallas, le rap, ou les films de Disney au Québec, en France, au Kenya ou au Japon. L'énonciation de ces produits culturels est identique partout, mais leur saisie est filtrée par le contexte de réception local ${ }^{12}$. On a plus de mal à admettre ce type de mécanisme pour les objets mondialisés, car la reconnaissance de la dimension sémiotique des objets est certainement plus récente et moins évidente. Or, ces phénomènes, sans être identiques, sont parfaitement comparables. Ce n'est pas parce qu'on porte les mêmes chaussures en Allemagne et en Californie ou qu'on mange les mêmes hamburgers au Brésil et à New York qu'on partage nécessairement les mêmes valeurs et qu'on est devenus identiques.

La saisie de ces objets, leur inscription dans un contexte local, leur adaptation contingente aux modes de vie du cru sont partie intégrante de leur 
fonctionnement sémiotique. L'appropriation de ces objets passe nécessairement par un processus d'adaptation, de filtrage, de négociation qui permet d'ancrer l'objet dans les pratiques de vie et les usages quotidiens. Il s'agit, pour reprendre la célèbre notion lévi-straussienne, d'un bricolage sémiotique et socioculturel permanent. À l'issue de ce procès, l'objet mondialisé a été modifié, redéfini, réinterprété, apprivoisé. Il ne ressemble plus que de loin, d'un point de vue sémiotique, à l'objet d'avant sa projection dans un contexte énonciatif local et concret.

Mais il ne faudrait pas oublier que l'intérêt même de ces objets est de ne pas appartenir, originairement, au contexte local. Le pouvoir de séduction des objets mondialisés consiste précisément dans leur altérité, tant spatiale (ils nous viennent toujours d'un ailleurs valorisé) que temporelle (ils nous font toujours miroiter une modernité, un futur valorisé). Et c'est précisément dans cette dialectique que se situent leur spécificité et leur intérêt pour l'analyste. Résumons donc les deux pôles de cette dialectique. D'une part, pour qu'un objet puisse aspirer à la mondialisation, il faut qu'il soit capable de fonctionner dans des contextes très différents, il doit être acontextuel, ou au moins transcontextuel. Ce qui explique pourquoi les boules de pétanque ou la "poutine» ne sont pas des objets mondialisés, alors que les hamburgers et les chaussures de sport le sont. D'autre part, une fois projetés dans un univers de réception concret, ces objets sont nécessairement soumis à un processus de saisie sémiotique, sont donc bricolés et recontextualisés selon des logiques et des conditions qui sont par définition variables.

L'analyse des «objets sans frontières» et de la dialectique d'interaction et de renforcement mutuel qui, autour d'eux, s'établit entre monde possible et monde réel montre par conséquent, de manière très claire à notre sens, que la mondialisation, loin d'être exclusivement une question de finance et de commerce, engage aussi, et peut-être avant tout, la dimension de l'imaginaire et des représentations.

\section{N O TES}

1. R. Barthes, Mythologies, Paris, Seuil, 1957; L'Aventure sémiologique, Paris, Seuil, 1985.

2. J. Baudrillard, Le Système des objets, Paris, Gallimard, 1968.

3. A. J. Greimas, Sémantique structurale, Paris, Larousse, 1966; Sémiotique et sciences sociales, Paris, Seuil, 1976.

4. A. Semprini, L'Objet comme procès et comme action, Paris, L'Harmattan, 1995.

5. H. Garfinkel, Ethnomethodological Studies, New York, Prentice Hall, 1967.

6. B. Latour, La Clef de Berlin, Paris, La Découverte, 1993.

7. E. Landowski et J.L. Fiorin, O gosto da gente, o gosto das coisas, Abordagem semiotica, Sao Paulo, Educ, 1997 (trad. ital., Gusti e disgusti, Sociosemiotica del quotidiano, Milano, Testo e immagine, 2000).

8. G. Marrone, C'era una volta il telefonino, Rome, Meltemi, 1999.

9. A. Semprini (sous la dir. de), Il senso delle cose, Milan, Franco Angeli, 1999.

10. C. Lévi-Strauss, dans la Pensée Sauvage (Paris, Plon, 1962), parle d'efficacité symbolique.

11. G. Leclerc, La Mondialisation culturelle, Paris, P.U.F., 2000.

12. A. Semprini, CNN et la mondialisation de l'imaginaire, Paris, Éd. du CNRS, 2000. 